\title{
Dynamic analysis of a SIQR epidemic model considering the interaction of environmental differences
}

\author{
Mingjian Wang ${ }^{1} \cdot$ Yuhan $\mathrm{Hu}^{1} \cdot$ Libing $\mathrm{Wu}^{1}$
}

Received: 14 June 2021 / Revised: 23 August 2021 / Accepted: 4 September 2021 /

Published online: 15 September 2021

C) Korean Society for Informatics and Computational Applied Mathematics 2021

\begin{abstract}
Infectious diseases have a devastating impact on individual health and social development. Different external environmental factors will affect the scale and the speed of disease outbreaks, such as sanitary conditions and policy interventions. In this paper, we attempt to establish a dual-system susceptible-infectious-quarantine-recovered model with different environmental impacts to explore it. For the deterministic model, we calculate the basic reproduction number, simultaneously, and investigate the local asymptotic stability of the disease-free equilibrium and the endemic equilibrium. Numerical simulations and theoretical analyses verify the conclusion of this paper. It is a surprise that there is a great probability of finding a quarantine inflection point, which can effectively control the scale of infection outbreak when two different systems of infection rate and recovery rate are determined.
\end{abstract}

Keywords SIQR epidemic model · Dual-system · Environmental differences · Interaction $\cdot$ Stability analysis

Mathematics Subject Classification 92D30, 34D20, 34A12

\section{Introduction}

Infectious diseases are caused by various pathogenic microorganisms (such as bacteria, viruses, spirochetes, parasites, etc.) that are infectious and can be prevalent in the population. For a long time, the spread and prevalence of infectious diseases have brought serious threats to human health, social and economic development. For example, the ravages of smallpox in the nineteenth century led to death of nearly 200 million people. In the 100 years of the twentieth century, the number of deaths from infec-

Libing Wu

beyondwlb@163.com

1 School of Science, University of Science and Technology Liaoning, Anshan 114051,

People's Republic of China 
tious diseases reached 1.68 billion, which was 13 times the number of deaths caused by multiple large-scale wars such as World War I and World War II. In November 2019, the COVID-19 spread globally. As of the end of February 2021, the number of infections has exceeded 100 million, and the death toll has mounted to 2.479 million [1]. Regrettably, it has limited the development of today's society and caused panic among the whole population in the world.

Mathematical model plays an important role in the study of infectious disease dynamics. It can be used to study the disease transmission principium, reveal its transmission laws and predict its development trend by quantitative analyses and numerical simulations [2,3]. In 1906, Hamer [4] analyzed a discrete-time model in order to understand the structure of repeated measles epidemics. In 1911, Ross [5] used the differential equation model to study the dynamic behavior of malaria transmission between mosquitoes and humans. At the beginning of the twentieth century, McKendrick and Kermack [6] analyzed the data that the plague outbreak affected the number and the survival days of patients from a perspective of mathematical point, and proposed a SIR infectious disease model. A "threshold theory" was established to judge the prevalence of the disease. Since then, mathematical modeling and research on the dynamics of infectious diseases have begun to develop rapidly, and many scholars have made a lot of progress in this area [7-9].

For centuries, quarantine has been used to limit the spread of infectious diseases, such as leprosy, plague, cholera, smallpox and influenza etc. [10-15]. Quarantine has also been used to control the COVID-19 which is spreading recently all over the world. On the basis of the SIR model proposed by K-M, Hethcote et al. [16,17] further subdivided the infected population into the non-quarantined infected and the quarantined infected, using $Q(t)$ to represent the number of quarantined people due to infection at time $t$, and established an infectious disease model with isolation. Assuming that the quarantined people have the corresponding immunity after recovery, the infectious disease model at this time is the SIQR model. Such as, Ma et al. [18] considered a SIQR epidemic model with standard incidence. Chahrazed [19] considered stability of a delayed SIQRS model with temporary immunity. Joshi et al. [20] studied a SIQR epidemic model with saturated incidence rate and proved the global stability of the disease-free and endemic equilibrium. On the other hand, May [21] implied the parameters in the model will be affected by various factors in the environment (temperature, humidity, rainfall, etc.), and variable environmental factors affect the dynamic behavior of the system all the time. Meanwhile, Zhang et al. [22] incorporated both the effects of immigration and nonlinear incidence, based on which they formulate a SEIR epidemic model. And Liu et al. [23-25] considered a multigroup epidemic model with different factors. Hattaf proposed a new definition of fractional derivatives and applied it to COVID-19 [26]. In another article, Hattaf combined the two modes of propagation to study the dynamic behavior of the model, and further proposed the biological analysis results [27]. Further, Hattaf combined the different transmission routes of COVID-19 and took into account the eect of awareness programs by media on the spread of this dangerous disease [28].

In real biological systems, some important parameters of epidemic models are always disturbed by external environmental mechanisms [29]. Obviously, it is more reasonable to consider dividing the whole into multigroups. However, we found that 
in previous studies, most models only considered the nature of multiple infectious diseases, and did not consider the different environmental interactions. In fact, the different risk of disease transmission results from different climates, diverse regions, and various living habits of human beings, and individuals in one environment can also affect others. For example, due to population movements (university opening and holidays, Chinese New Year travel, etc.), infected individuals by the current COVID19 will flow from one environment to another environment, then it's hard to predict the impact on the current environment. In this article, we reformulate the existing SIQR model, redefine the infection and quarantine area, describe the impact of different environmental conditions, and prove its stability. Numerical simulations verify its correctness.

The paper is organized as follows. In Sect. 2, we establish a SIQR model that considers the mutual influence of two environments and has isolation measures, and boundedness of the solutions is obtained. In Sect. 3, we obtain the basic reproduction number, disease-free equilibrium and endemic disease equilibrium of the model. We prove the local asymptotic stability and the global asymptotic stability conditions of the equilibrium. In Sect. 4, the numerical simulations of our model are performed to confirm and illustrate our results. In Sect. 5, we draw conclusions and indicate some future works.

\section{Model description}

In this section, the total population size changes with time $t$ and we define it $N(t)$. Different environmental conditions will affect the infection rate and recovery rate of the disease. Groups with better environmental conditions have lower infection rates and higher recovery rates, while groups with worse environmental conditions have the opposite result. Based on this situation, we divide the total population size into two parts. $N_{1}(t)$ represents the group with better environmental conditions, and $N_{2}(t)$ represents another group with worse environmental conditions, so there is $N=$ $N_{1}+N_{2}$. The whole population is divided into four groups: Susceptible(S), Infected(I), Quarantined/isolation(Q), Recovered(R), with sizes denoted by $S(t), I(t), Q(t), R(t)$, respectively.

We assumed that the susceptible population has a constant input rate $\Lambda$ which is named as the susceptible recruitment rate corresponding to births and immigration. And the people entered into the environment at the $\Lambda$ are entered into separate groups at different rates, respectively represented by $\Lambda_{1}$ and $\Lambda_{2}$, so there is $\Lambda=\Lambda_{1}+\Lambda_{2}$. Each group has a same emigration rate which is denoted as the natural death rate $\mu$.

Here, without loss of generality, we only consider the one way that human contact to spread diseases. Therefore, we consider the spread of the disease through contact between the infected individual and the susceptible individual. When a susceptible individual comes into contact with an infected individual, he/she will become an infected individual with a certain probability according to his/her own immunity. This standard incidence rate is represented by $\frac{\beta S I}{N}, \beta$ represents the transmission rate of the disease. 


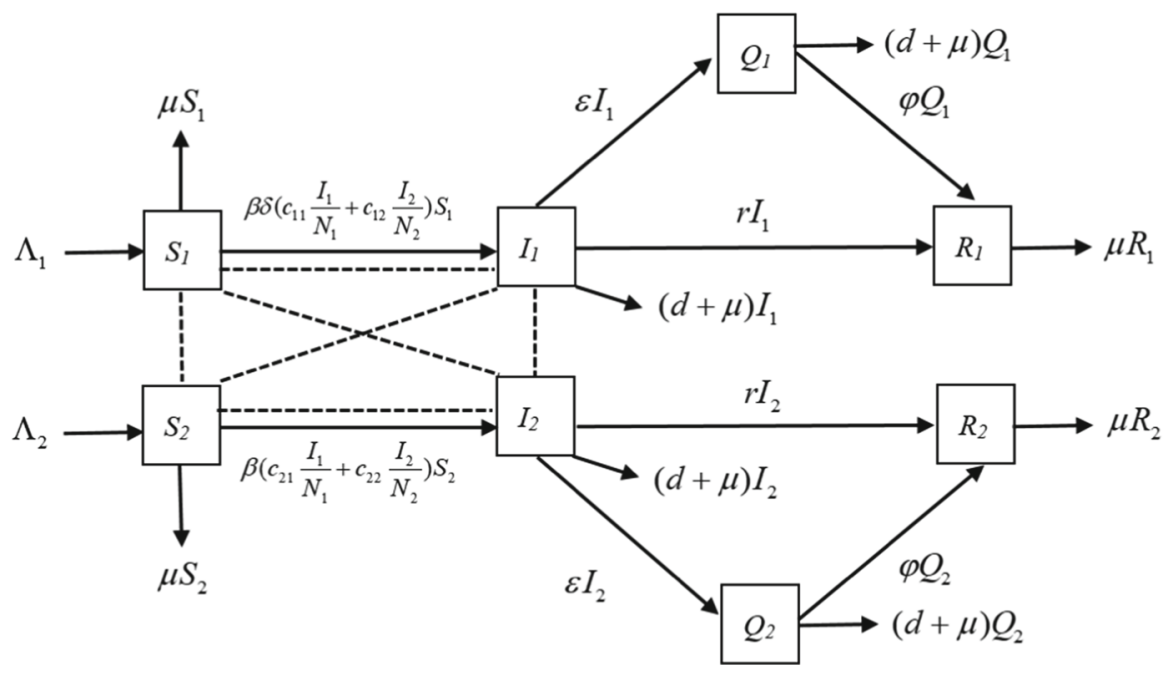

Fig. 1 The flow diagram of the model

In contemporary times, the government reduces the contact between infected and susceptible individuals through various methods such as family isolation, hospital isolation, city closures, and travel restrictions, etc. We describe this phenomenon by establishing isolation (Q). When infected individuals are diagnosed as having been infected, they will be moved into isolation (Q) for separate treatment, $\varepsilon$ stands for the isolation rate. Meanwhile, we define $\varphi$ as the cure rate related to isolation, $r$ stands for the cure rate related to infected, $d$ stands for the disease-related death rate. A better environmental (group 1) condition will inhibit the probability of the spread of the disease. On the contrary, a worse environment (group 2) condition will increase the probability of the spread of the disease.

We denote $\delta$ to represent the contagious reduction rate, and the better environment reduces the infection rate by $\delta$ times than a group with the worse environment, where $\delta \in(0,1]$. Therefore, the infection rate of a group with a worse environment is defined as $\beta$, and the infection rate of a group with a better environment is defined as $\delta \beta$. In addition, due to the influence of objective factors such as family, work and living conditions. An individual's living environment is relatively fixed and can hardly be changed in a short period of time. Even if an individual occasionally leaves the current environment, he or she will return soon afterwards. Therefore, we assume that the change of an individual leaving the current environment to another environment is negligible. However, individuals in different environments will also come into contact with each other [30]. $c_{i j}$ is denoted as the contact ratio of the $i$ group members to the $j$ group members, where $c_{i j}=\frac{N_{j}}{N_{i}+N_{j}}$ and $i, j=1,2$.

All rates are positive constants.

Based on the above assumptions and condition of hypothesis, we draw a model flow diagram as is shown in Fig. 1. 
Table 1 The notations of the model

\begin{tabular}{ll}
\hline Notation & Description \\
\hline$S_{i}(t)$ & The number of susceptible individuals in group $i$ at time $t$ \\
$I_{i}(t)$ & The number of infectious individuals in group $i$ at time $t$ \\
$Q_{i}(t)$ & The number of isolated (quarantined) individuals in group $i$ at time $t$ \\
$R_{i}(t)$ & The number of recovered individuals in group $i$ at time $t$ \\
$\Lambda_{i}$ & The birth and immigration rate in group $i$ \\
$\mu$ & The natural death rate \\
$\beta$ & The transmission rate of the disease \\
$d$ & The disease-related death rate \\
$\varepsilon$ & The isolation rate \\
$r$ & The cure rate related to infected \\
$\varphi$ & The cure rate related to isolation \\
$\delta$ & The contagious reduction rate \\
$c_{i j}$ & The contact ratio of members of group $i$ to members of group $j$ \\
\hline
\end{tabular}

The meaning of each symbol in Fig. 1 is shown in Table 1. As shown in Fig. 1, the states of susceptible, infected, isolation, recovered are as follows. In the unit time, there is the number of $\Lambda$ into the susceptible individual. When a susceptible individual $\left(S_{1}, S_{2}\right)$ and an infected individual $\left(I_{1}, I_{2}\right)$ come into contact in a mixed proportion, they will be infected with a probability of $\beta$. Meanwhile, a better environment will correspondingly reduce the risk of infection by $\delta$ times, and members of $\mu S$ will naturally die. So the number of the susceptible individual per unit time is $\Lambda_{i}-\beta \delta\left(c_{i j} \frac{I_{i}}{N_{i}}+c_{i j} \frac{I_{j}}{N_{j}}\right) S_{i}-\mu S_{i}$.

In unit time, people who have moved into the infected individual will leave the current class in three ways: Firstly, if the infection is found, infected individual will be moved into the isolation class at a ratio of $\varepsilon$; Secondly, some self-healing in the infected will be moved into the immune class at a ratio of $r$; Thirdly, the individual which dead due to illness and naturaly dead in the infected will be moved out the system in a ratio of $d$ and $\mu$. So the number of the infected individual per unit time is $\beta \delta_{i}\left(c_{i j} \frac{I_{i}}{N_{i}}+c_{i j} \frac{I_{j}}{N_{j}}\right) S_{i}-(r+\varepsilon+\mu+d) I_{i}$.

Similarly, the isolation individual will be taken out of the system in the way of death for illness or natural death, or be cured into immune class. So the number of the isolation individual per unit time is $\varepsilon I_{i}-(\varphi+d+\mu) Q_{i}$. And the recovered individuals will be removed from the system in the way of natural death. So the number of the recovered individuals per unit time is $r I_{i}+\varphi Q_{i}-\mu R_{i}$. 
Therefore, based on the above discussion, the SIQR epidemic model with different environmental impacts is established as follows:

$$
\begin{aligned}
& \left\{\begin{array}{l}
\frac{d S_{1}}{d t}=\Lambda_{1}-\beta \delta\left(c_{11} \frac{I_{1}}{N_{1}}+c_{12} \frac{I_{2}}{N_{2}}\right) S_{1}-\mu S_{1} \\
\frac{d I_{1}}{d t}=\beta \delta\left(c_{11} \frac{I_{1}}{N_{1}}+c_{12} \frac{I_{2}}{N_{2}}\right) S_{1}-(r+\varepsilon+\mu+d) I_{1} \\
\frac{d Q_{1}}{d t}=\varepsilon I_{1}-(\varphi+d+\mu) Q_{1} \\
\frac{d R_{1}}{d t}=r I_{1}+\varphi Q_{1}-\mu R_{1}
\end{array}\right. \\
& \begin{cases}\frac{d S_{2}}{d t}=\Lambda_{2}-\beta\left(c_{21} \frac{I_{1}}{N_{1}}+c_{22} \frac{I_{2}}{N_{2}}\right) S_{2}-\mu S_{2} \\
\frac{d I_{2}}{d t}=\beta\left(c_{21} \frac{I_{1}}{N_{1}}+c_{22} \frac{I_{2}}{N_{2}}\right) S_{2}-(r+\varepsilon+\mu+d) I_{2} \\
\frac{d Q_{2}}{d t}=\varepsilon I_{2}-(\varphi+d+\mu) Q_{2} \\
\frac{d R_{2}}{d t}=r I_{2}+\varphi Q_{2}-\mu R_{2}\end{cases}
\end{aligned}
$$

where $\Lambda>0, \beta>0, \mu>0, \delta \in(0,1], \varphi, \varepsilon, r \in(0,1)$. And there is $S(t)+I(t)+$ $Q(t)+R(t)=N$ at any time t.

It easy to know that $\frac{d N(t)}{d t}=\Lambda-\mu N$, so $N(t)=\left(N_{0}-\frac{\Lambda}{\mu}\right) e^{-\mu t}+\frac{\Lambda}{\mu}$, where $N_{0}=N(0)$, and then $\lim _{t \rightarrow \infty} N(t)=\frac{\Lambda}{\mu}$. The positive invariant set of system (1) is:

$$
\Gamma=\left\{(S, I, Q, R) \in R_{4}^{+}: S+I+Q+R \leq \frac{\Lambda}{\mu}\right\} .
$$

\section{Stability analysis}

\subsection{Equilibria and the basic reproduction number}

For a population dynamics system, studying its equilibrium points and the basic reproduction number is the precondition for predicting the development trend of populations within the system.

$$
\begin{aligned}
& \left\{\begin{array}{l}
\frac{d S_{1}}{d t}=\Lambda_{1}-\beta \delta\left(c_{11} \frac{I_{1}}{N_{1}}+c_{12} \frac{I_{2}}{N_{2}}\right) S_{1}-\mu S_{1} \\
\frac{d I_{1}}{d t}=\beta \delta\left(c_{11} \frac{I_{1}}{N_{1}}+c_{12} \frac{I_{2}}{N_{2}}\right) S_{1}-(r+\varepsilon+\mu+d) I_{1} \\
\frac{d Q_{1}}{d t}=\varepsilon I_{1}-(\varphi+d+\mu) Q_{1}
\end{array}\right. \\
& \left\{\begin{array}{l}
\frac{d S_{2}}{d t}=\Lambda_{2}-\beta\left(c_{21} \frac{I_{1}}{N_{1}}+c_{22} \frac{I_{2}}{N_{2}}\right) S_{2}-\mu S_{2} \\
\frac{d I_{2}}{d t}=\beta\left(c_{21} \frac{I_{1}}{N_{1}}+c_{22} \frac{I_{2}}{N_{2}}\right) S_{2}-(r+\varepsilon+\mu+d) I_{2} \\
\frac{d Q_{2}}{d t}=\varepsilon I_{2}-(\varphi+d+\mu) Q_{2}
\end{array}\right.
\end{aligned}
$$

Theorem 1 In system (2), when the basic reproduction number $R_{0}<1$, there is a disease-free equilibrium, i.e.,

$$
P^{0}=\left(S_{1}^{0}, I_{1}^{0}, Q_{1}^{0}, S_{2}^{0}, I_{2}^{0}, Q_{2}^{0}\right)=\left(\frac{\Lambda_{1}}{\mu}, 0,0, \frac{\Lambda_{2}}{\mu}, 0,0\right) .
$$


When the basic reproduction number $R_{0}>1$ and $\mu \omega+\frac{\Lambda_{2} \beta}{\Lambda}-\frac{\Lambda_{1} \delta \beta}{\Lambda}<0$, there is one endemic equilibrium, i.e.,

$$
\begin{aligned}
& P^{*}=\left(S_{1}^{*}, I_{1}^{*}, Q_{1}^{*}, S_{2}^{*}, I_{2}^{*}, Q_{2}^{*}\right)=\left(\frac{\omega \Lambda_{1}}{\beta \delta \mu+\omega \mu(1-\delta)}, \frac{\beta \delta I^{*} S_{1}^{*}}{\omega\left(N_{1}^{*}+N_{2}^{*}\right)}, \frac{\varepsilon I_{1}^{*}}{\omega-r}, \frac{\omega \Lambda_{2}}{\beta \delta \mu+\omega \mu(1-\delta)},\right. \\
& \left.\frac{\beta \delta I^{*} S_{2}^{*}}{\omega\left(N_{1}^{*}+N_{2}^{*}\right)}, \frac{\varepsilon I_{2}^{*}}{\omega-r}\right) . \text { where } I^{*}=\frac{\Lambda \delta \eta-(1+\delta) \mu \omega \pm\left[\Lambda^{2} \delta^{2} \eta^{2}+(\delta-1)^{2} \mu^{2} \omega^{2}+2 \eta \mu \omega\left(\delta^{2}-\delta\right)\left(\Lambda_{1}-\Lambda_{2}\right)\right]^{\frac{1}{2}}}{2 \delta \omega}, \\
& \omega=r+\varepsilon+\mu+d .
\end{aligned}
$$

Proof On putting the derivatives in the left-hand side of system (2) equal zero and we get that the model has a disease-free equilibrium, where $I_{1}^{0}=I_{2}^{0}=0, Q_{1}^{0}=Q_{2}^{0}=0$. $P^{0}=\left(S_{1}^{0}, I_{1}^{0}, Q_{1}^{0}, S_{2}^{0}, I_{2}^{0}, Q_{2}^{0}\right)=\left(\frac{\Lambda_{1}}{\mu}, 0,0, \frac{\Lambda_{2}}{\mu}, 0,0\right)$

In the presence of disease, we can get the unique disease-endemic equilibrium, where $I \neq 0, \eta=\frac{\beta}{N}$.

$$
\begin{aligned}
& P^{*}=\left(S_{1}^{*}, I_{1}^{*}, Q_{1}^{*}, S_{2}^{*}, I_{2}^{*}, Q_{2}^{*}\right)=\left(\frac{\omega \Lambda_{1}}{\beta \delta \mu+\omega \mu(1-\delta)}, \frac{\beta \delta I^{*} S_{1}^{*}}{\omega\left(N_{1}^{*}+N_{2}^{*}\right)}, \frac{\varepsilon I_{1}^{*}}{\omega-r}, \frac{\omega \Lambda_{2}}{\beta \delta \mu+\omega \mu(1-\delta)},\right. \\
& \left.\frac{\beta \delta I^{*} S_{2}^{*}}{\omega\left(N_{1}^{*}+N_{2}^{*}\right)}, \frac{\varepsilon I_{2}^{*}}{\omega-r}\right) . \text { We can get }
\end{aligned}
$$

$$
I^{*}=I_{1}^{*}+I_{2}^{*}=\frac{\delta \eta I^{*} S_{1}^{*}+\eta I S_{2}^{*}}{\omega}
$$

and

$$
\omega=\delta \eta S_{1}^{*}+\eta S_{2}^{*}
$$

Then according to Eqs. (3) and (4), we have

$$
\frac{\delta \Lambda_{1}}{\delta \eta I^{*}+\mu}+\frac{\Lambda_{2}}{\eta I^{*}+\mu}=\frac{\omega}{\eta}
$$

which is

$$
\delta \Lambda_{1}\left(\eta I^{*}+\mu\right)+\Lambda_{2}\left(\delta \eta I^{*}+\mu\right)=\frac{\omega}{\eta}\left(\eta I^{*}+\mu\right)\left(\delta \eta I^{*}+\mu\right)
$$

Solve the Eq. (6) and discriminant of root, there is at least one positive definite solution when $\mu \omega+\frac{\Lambda_{2} \beta}{\Lambda}-\frac{\Lambda_{1} \delta \beta}{\Lambda}<0$. And we have $I^{*}=$ $\frac{\Lambda \delta \eta-(1+\delta) \mu \omega \pm\left[\Lambda^{2} \delta^{2} \eta^{2}+(\delta-1)^{2} \mu^{2} \omega^{2}+2 \eta \mu \omega\left(\delta^{2}-\delta\right)\left(\Lambda_{1}-\Lambda_{2}\right)\right]^{\frac{1}{2}}}{2 \delta \omega}$. This completes the proof.

Then, we use the next generation matrix to get the basic reproduction number $R_{0}$ of the SIQR model. According to the inference of Theorem 1, we have got the disease-free equilibrium, $P^{0}=\left(S_{1}^{0}, I_{1}^{0}, Q_{1}^{0}, S_{2}^{0}, I_{2}^{0}, Q_{2}^{0}\right)=\left(N_{1}^{0}, 0,0, N_{2}^{0}, 0,0\right)$.

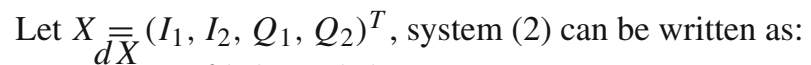

$$
\begin{aligned}
& \frac{d X}{d t}=f(X)-v(X) \\
& f=\left(\begin{array}{c}
\beta \delta\left(c_{11} \frac{I_{1}}{N_{1}}+c_{12} \frac{I_{2}}{N_{2}}\right) S_{1} \\
\beta\left(c_{21} \frac{I_{1}}{N_{1}}+c_{22} \frac{I_{2}}{N_{2}}\right) S_{2} \\
0 \\
0
\end{array}\right), v=\left(\begin{array}{c}
\left(r_{1}+\varepsilon+\mu+d\right) I_{1} \\
\left(r_{2}+\varepsilon+\mu+d\right) I_{2} \\
-\varepsilon I_{1}+(\varphi+d+\mu) Q_{1} \\
-\varepsilon I_{2}+(\varphi+d+\mu) Q_{2}
\end{array}\right)
\end{aligned}
$$


Further, taking the derivatives of $f, v$ with respected to infected compartments $I_{1}, I_{2}, Q_{1}, Q_{2}$ at the disease-free equilibrium $P^{0}$, respectively, we have

$$
\begin{aligned}
F & =\left(\begin{array}{cccc}
\beta \delta c_{11} & \beta \delta c_{12} \frac{N_{1}}{N_{2}} & 0 & 0 \\
\beta c_{21} \frac{N_{1}}{N_{2}} & \beta c_{22} & 0 & 0 \\
0 & 0 & 0 & 0 \\
0 & 0 & 0 & 0
\end{array}\right) \\
V & =\left(\begin{array}{cccc}
r_{1}+\varepsilon+\mu+d & 0 & 0 & 0 \\
0 & r_{2}+\varepsilon+\mu+d & 0 & 0 \\
-\varepsilon & 0 & \varphi+d+\mu & 0 \\
0 & -\varepsilon & 0 & \varphi+d+\mu
\end{array}\right)
\end{aligned}
$$

Then, the next generation matrix can be expressed as

$$
K=F V^{-1}=\left(\begin{array}{cccc}
\frac{\beta \delta c_{11}}{r_{1}+\varepsilon+\mu+d} & \frac{\beta \delta c_{12} \frac{N_{1}}{N_{2}}}{r_{2}+\varepsilon+\mu+d} & 0 & 0 \\
\frac{\beta c_{21} \frac{N_{1}}{N_{2}}}{r_{1}+\varepsilon+\mu+d} & \frac{\beta c_{22}}{r_{2}+\varepsilon+\mu+d} & 0 & 0 \\
0 & 0 & 0 & 0 \\
0 & 0 & 0 & 0
\end{array}\right)
$$

Hence, the basic reproduction number of system (2) is the spectral radius of matrix (10) as follows:

$$
R_{0}=\rho(K)=\frac{\beta\left(c_{22}+\delta c_{11}\right)}{d+\varepsilon+\mu+r_{2}}
$$

\subsection{Stability of the disease-free equilibrium}

Theorem 2 The disease-free equilibrium is local asymptotically stable if $d+\varepsilon+\mu+$ $r>\beta$.

Proof Plug $\left(S_{1}^{0}, I_{1}^{0}, Q_{1}^{0}, S_{2}^{0}, I_{2}^{0}, Q_{2}^{0}\right)=\left(N_{1}^{0}, 0,0, N_{2}^{0}, 0,0\right)$ into the formula, we can get the Jacobin matrix about $P^{0}$ is

$$
J\left(P^{0}\right)=\left(\begin{array}{cccccc}
-\mu & 0 & -\beta \delta c_{11} & -\beta \delta c_{12} \frac{N_{1}^{0}}{N_{2}^{0}} & 0 & 0 \\
0 & -\mu & -\beta c_{21} \frac{N_{2}^{0}}{N_{1}^{0}} & -\beta c_{22} & 0 & 0 \\
0 & 0 & \beta \delta c_{11}-\omega & \beta \delta c_{12} \frac{N_{1}^{0}}{N_{2}^{0}} & 0 & 0 \\
0 & 0 & \beta c_{21} \frac{N_{2}^{0}}{N_{1}^{0}} & \beta c_{22}-\omega & 0 & 0 \\
0 & 0 & \varepsilon & 0 & -(\varphi+d+\mu) & 0 \\
0 & 0 & 0 & \varepsilon & 0 & -(\varphi+d+\mu)
\end{array}\right)
$$


The characteristic equation of the above matrix is

$$
\begin{aligned}
& |\lambda E-J| \\
& =\left|\begin{array}{cccccc}
\lambda+\mu & 0 & \beta \delta c_{11} & \beta \delta c_{12} \frac{N_{1}^{0}}{N_{2}^{0}} & 0 & 0 \\
0 & \lambda+\mu & \beta c_{21} \frac{N_{2}^{0}}{N_{1}^{0}} & \beta c_{22} & 0 & 0 \\
0 & 0 & \lambda-\beta \delta c_{11}+\omega & -\beta \delta c_{12} \frac{N_{1}^{0}}{N_{2}^{0}} & 0 & 0 \\
0 & 0 & -\beta c_{21} \frac{N_{2}^{0}}{N_{1}^{0}} & \lambda-\beta c_{22}+\omega & 0 & 0 \\
0 & 0 & -\varepsilon & 0 & \lambda+\omega-r & 0 \\
0 & 0 & 0 & -\varepsilon & 0 & \lambda+\omega-r
\end{array}\right| \\
& =0
\end{aligned}
$$

Then solve the characteristic equation of (13) is

$$
(\lambda+\mu)^{2}[\lambda+(\varphi+d+\mu)]^{2}\left[\lambda^{2}-\lambda\left(\delta \eta N_{1}^{0}+\eta N_{2}^{0}-2 \omega\right)-\left(\delta \eta N_{1}^{0} \omega+\eta N_{2}^{0} \omega\right)+\omega^{2}\right]=0
$$

It is easy to obtain that the eigenvalue of the Eq. (14) is $\lambda_{1,2}=-\mu<0, \lambda_{3,4}=$ $-(\varphi+d+\mu)<0$. We consider the first method of Lyapunov and following the Veda's Theorem

$$
\left\{\begin{array}{l}
\lambda_{5}+\lambda_{6}=\delta \eta N_{1}^{0}+\eta N_{2}^{0}-2 \omega<0 \\
\lambda_{5} \lambda_{6}=\omega^{2}-\delta \eta N_{1}^{0} \omega-\eta N_{2}^{0} \omega>0
\end{array}\right.
$$

By solving the inequality (15), we get $\omega-\frac{\beta \delta N_{1}^{0}+\beta N_{2}^{0}}{N_{1}^{0}+N_{2}^{0}}>0$ if $\omega>\beta$.

While, all roots of the characteristic equation of the Jacobin matrix $J\left(P^{0}\right)$ have negative real parts. The system (2) is locally asymptotically stable at $P^{0}$. The proof is completed.

\subsection{Stability of the endemic disease equilibrium}

In this part, we investigate the stability of the endemic equilibrium, $P^{*}=$ $\left(\frac{\Lambda_{1}}{\delta \eta I^{*}+\mu}, \frac{\Lambda_{2}}{\eta I^{*}+\mu}, \frac{\delta \eta I^{*} S_{1}^{*}}{\omega}, \frac{\eta I^{*} S_{2}^{*}}{\omega}, \frac{\varepsilon I_{1}^{*}}{\omega-r}, \frac{\varepsilon I_{2}^{*}}{\omega-r}\right)$.

Theorem 3 It is local asymptotically stable if the existence condition of endemic disease equilibrium is met. 
Proof If endemic equilibrium $\left(S_{1}^{*}, I_{1}^{*}, Q_{1}^{*}, S_{2}^{*}, I_{2}^{*}, Q_{2}^{*}\right)$ exists, we can get the Jacobin matrix about $P^{*}$ is

$$
\begin{aligned}
& J\left(P^{*}\right) \\
& =\left(\begin{array}{cccccc}
-\frac{\beta \delta}{N^{*}}\left(I_{1}^{*}+I_{2}^{*}\right)-\mu & 0 & -\frac{\beta \delta}{N^{*}} S_{1}^{*} & -\frac{\beta \delta}{N^{*}} S_{1}^{*} & 0 & 0 \\
0 & -\frac{\beta}{N^{*}}\left(I_{1}^{*}+I_{2}^{*}\right)-\mu & -\frac{\beta}{N^{*}} S_{2}^{*} & -\frac{\beta}{N^{*}} S_{2}^{*} & 0 & 0 \\
\frac{\beta \delta}{N^{*}}\left(I_{1}^{*}+I_{2}^{*}\right) & 0 & \frac{\beta \delta}{N^{*}} S_{1}^{*}-\omega & \frac{\beta \delta}{N^{*}} S_{1}^{*} & 0 & 0 \\
0 & \frac{\beta}{N^{*}}\left(I_{1}^{*}+I_{2}^{*}\right) & \frac{\beta}{N^{*}} S_{2}^{*} & \frac{\beta}{N^{*}} S_{2}^{*}-\omega & 0 & 0 \\
0 & 0 & \varepsilon & 0 & r-\omega & 0 \\
0 & 0 & 0 & \varepsilon & 0 & r-\omega
\end{array}\right)
\end{aligned}
$$

After simplification, the characteristic equation of the above matrix is

$$
=[\lambda+(\varphi+d+\mu)]^{2}\left|\begin{array}{cccc}
\lambda+\delta \eta I^{*}+\mu & 0 & \delta \eta S_{1}^{*} & \delta \eta S_{1}^{*} \\
0 & \lambda+\eta I^{*}+\mu & \eta S_{2}^{*} & \eta S_{2}^{*} \\
-\delta \eta I^{*} & 0 & \lambda-\delta \eta S_{1}^{*}+\omega & -\delta \eta S_{1}^{*} \\
0 & -\eta I^{*} & -\eta S_{2}^{*} & \lambda-\eta S_{2}^{*}+\omega
\end{array}\right|=0
$$

The result after determinant transformation is

$$
[\lambda+(\varphi+d+\mu)]^{2}(\lambda+\omega)\left|\begin{array}{ccc}
\lambda+\delta \eta I^{*}+\mu & 0 & \delta \eta S_{1}^{*} \\
0 & \lambda+\eta I^{*}+\mu & \eta S_{2}^{*} \\
\lambda+\mu & \lambda+\mu & \lambda+\omega
\end{array}\right|=0
$$

It leads to $\lambda_{1,2}=-(\varphi+d+\mu)<0, \lambda_{3}=-\omega<0$. Next, we analyse the determinant

$$
\left|\begin{array}{ccc}
\lambda+b_{1} & 0 & b_{3} \\
0 & \lambda+b_{2} & b_{4} \\
\lambda+\mu & \lambda+\mu & \lambda+\omega
\end{array}\right|=0
$$

where

$$
\left\{\begin{array}{c}
b_{1}=\delta \eta I^{*}+\mu>0 \\
b_{2}=\eta I^{*}+\mu>0 \\
b_{3}=\delta \eta S_{1}^{*}>0 \\
b_{4}=\eta S_{2}^{*}>0
\end{array}\right.
$$

This is

$$
\lambda^{3}+h_{1} \lambda^{2}+h_{2} \lambda+h_{3}=0
$$

For $P^{*}$ exists $I_{1}^{*}+I_{2}^{*}=I^{*}$, which is $\delta \eta I^{*} S_{1}^{*}+\eta I^{*} S_{2}^{*}=I^{*} \omega$. Remove $I^{*}$ to get $\delta \eta S_{1}^{*}+\eta S_{2}^{*}=\omega$.

By substituting the formula in to (15), we can get

$$
\left\{\begin{array}{l}
h_{1}=b_{1}+b_{2}>0 \\
h_{2}=b_{1} b_{3}+b_{1} b_{2}+b_{2} b_{4}-b_{3} \mu-b_{4} \mu>0 \\
h_{3}=b_{1} b_{2} \omega-b_{2} b_{3} \mu-b_{1} b_{4} \mu>0
\end{array}\right.
$$



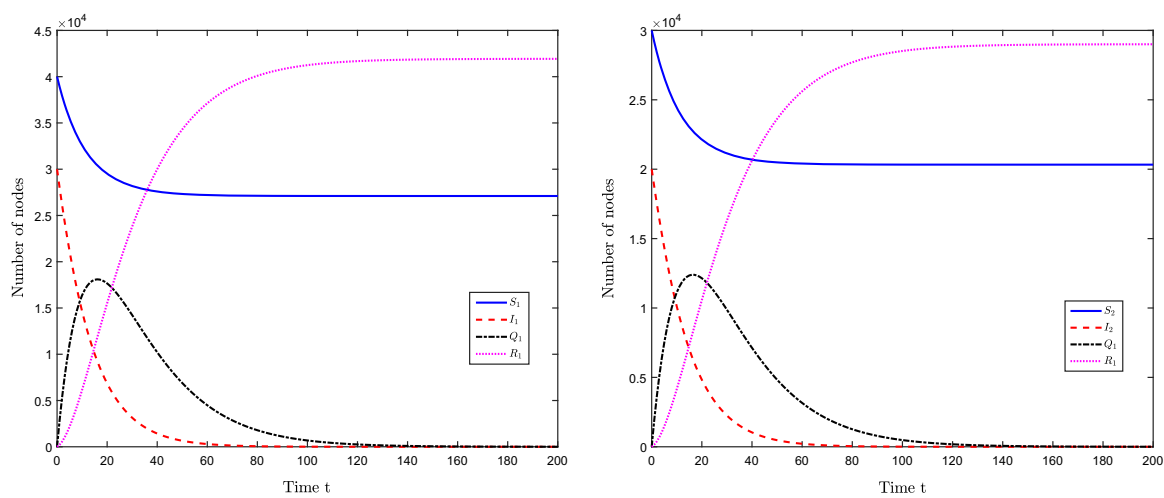

Fig. 2 Stability of the disease-free equilibrium

$$
\left\{\begin{array}{l}
h_{1}=\delta \eta I^{*}+\eta I^{*}+2 \mu>0 \\
h_{2}=\delta \eta I^{*} b_{3}+b_{1} b_{2}+\eta I^{*} b_{4}>0 \\
h_{3}=\delta \eta I^{*} b_{2} b_{3}+\eta I^{*} b_{1} b_{4}>0
\end{array}\right.
$$

Since Eqs. (20) and (23), we can obtain

$$
\begin{aligned}
& h_{1} h_{2}-h_{3} \\
& =\left(b_{1}+b_{2}\right)\left(b_{1} b_{3}+b_{1} b_{2}+b_{2} b_{4}-b_{3} \mu-b_{4} \mu\right)-\left[b_{1} b_{2}\left(b_{3}+b_{4}\right)-b_{2} b_{3} \mu-b_{1} b_{4} \mu\right] \\
& =b_{1}^{2} b_{3}+b_{1}^{2} b_{2}+b_{2}^{2} b_{1}+b_{2}^{2} b_{4}-b_{1} b_{3} \mu-b_{2} b_{4} \mu \\
& =b_{1} b_{3} \delta \eta I^{*}+b_{1}^{2} b_{2}+b_{2}^{2} b_{1}+b_{2} b_{4} \eta I^{*}>0
\end{aligned}
$$

While, all roots of the characteristic equation of the Jacobin matrix $J\left(P^{*}\right)$ have negative real parts. The system (2) is locally asymptotically stable at $P^{*}$. The proof is completed.

\section{Numerical simulation}

In this section, We will give some numerical simulations to illustrate the theoretical model and the results of the previous section. This example is inspired by real acute the early evolution of the COVID-19 in Brazil [31], with time unit of one day.

The initial conditions for the disease free equilibrium point are assumed as follows: $N_{1}(0)=70000, N_{2}(0)=50000, S_{1}(0)=40000, I_{1}(0)=30000, Q_{1}(0)=$ $0, R_{1}(0)=0, S_{2}(0)=30000, I_{2}(0)=20000, Q_{2}(0)=0, R_{2}(0)=0 . \quad$ In the first experiment, parameters are given as follows: $\beta=0.01, \delta=0.9, r=0.005, \varepsilon=$ $0.1, \varphi=0.05, d=0.0008, \Lambda_{1}=0.01, \Lambda_{2}=0.02, \mu=0.00003$ and it can be $g o t$ $R_{0}=0.6734<1$ according to Eq. (11). Theorems 1 and 2 indicate that the disease disappears gradually and the disease-free equilibrium is stable. Figure 2 further confirms this conclusion. 

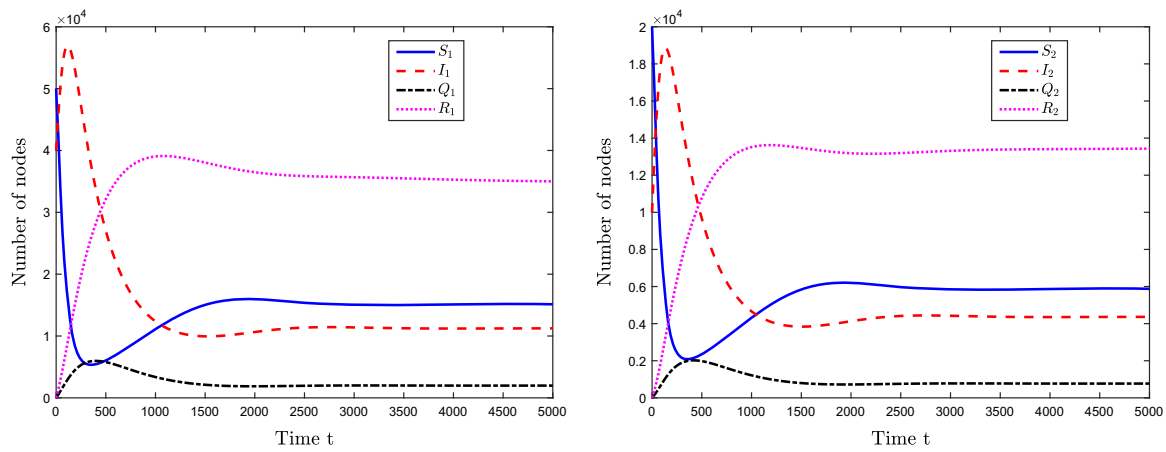

Fig. 3 Stability of the endemic disease equilibrium

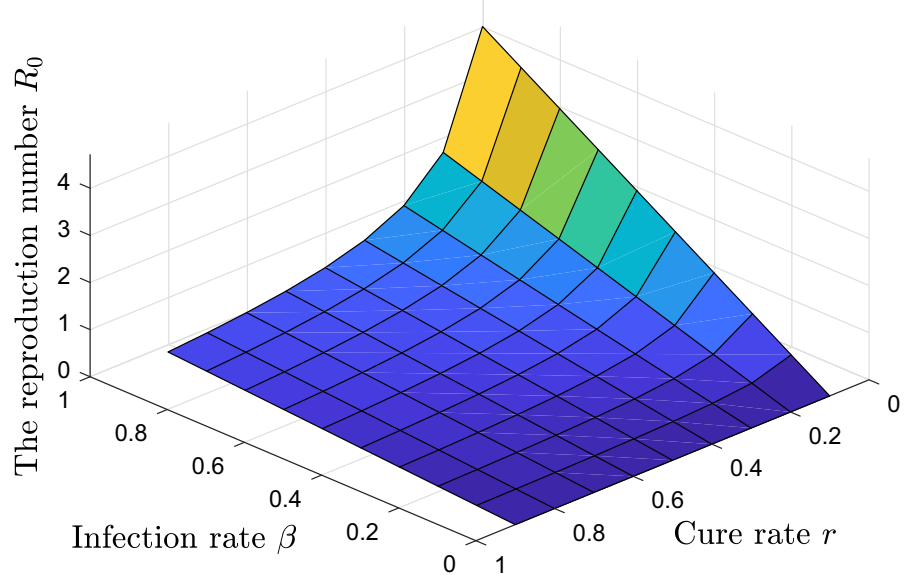

Fig. 4 The tendency of $R_{0}$ with $\beta$ and $r$

In the second experiment, parameters are given as follows: $\beta=0.01, \delta=0.2, r=$ $0.0015, \varepsilon=0.1, \varphi=0.002, d=0.0008, \Lambda_{1}=40.4, \Lambda_{2}=18.8, \mu=0.0006$ and it can be got $R_{0}=1.4129>1$ according to Eq. (11). Theorems 1 and 3 indicate that the disease is persistent and stable and the endemic disease equilibrium is stable. Figure 3 further confirms this conclusion.

To examine the sensitivity of the basic reproduction number $R_{0}$ to some parameters, we compute the derivatives of $R_{0}$ with respect to these parameters.

$$
\begin{gathered}
\frac{\partial R_{0}}{\partial \beta}=\frac{c_{22}+\delta c_{11}}{d+\varepsilon+\mu+r}>0 \\
\frac{\partial R_{0}}{\partial r}=-\frac{\beta\left(c_{22}+\delta c_{11}\right)}{(d+\varepsilon+\mu+r)^{2}}<0
\end{gathered}
$$

And a plot of $R_{0}$ as a function of $\beta$ and $r$ is shown in Fig. 4. From the results, it is clear that the $R_{0}$ increases as $\beta$ and the $R_{0}$ decreases as $r$ increase. The cure rate is directly related to the living environment and isolation conditions. An effective strategy to 


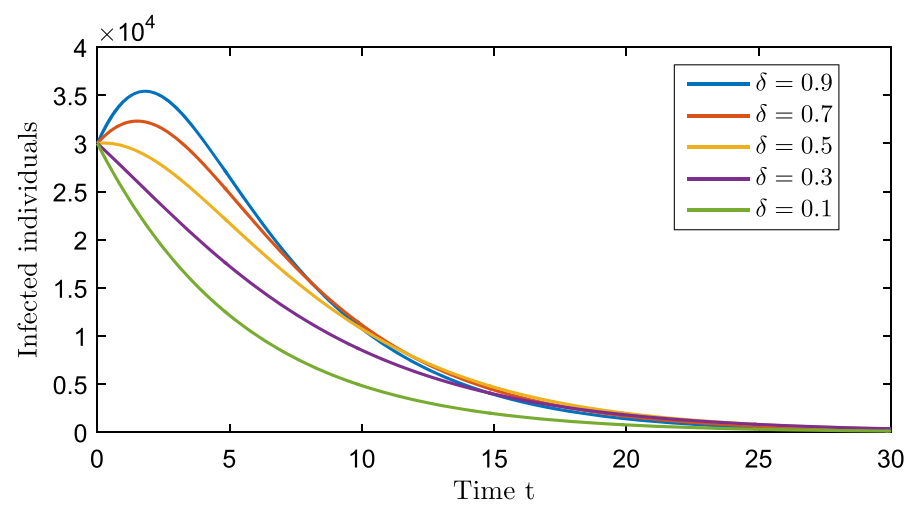

Fig. 5 The change of infected individuals in different reduction rates

control the scale of the disease is to control the basic reproduction number $R_{0}$ below one. Based on this principle, methods such as self-isolation and home isolation should be advocated, so as to avoid contacts between patients and susceptible individuals as much as possible. An environment with poor sanitary conditions is a breeding ground for viruses. When the overall environmental quality is improved, the spread of diseases will be effectively controlled.

Next, we consider that different environmental factors will affect the current system, and parameters are given as follows: $\beta=0.048, r=0.125, \varepsilon=0.09, \varphi=$ $0.0125, d=0.001, \Lambda_{1}=0.012, \Lambda_{2}=0.024, \mu=0.0003$. Figure 5 represents the image of changes in the number of infected people reduced in different proportions due to environmental impacts. The number of infections will decrease as the contagious reduction rate $\delta$ decreased and the maximum number of infections will gradually decrease. When $\delta$ is greater than 0.5 , the number of infected individuals increased first and then decreased because of the high infection rate in the population. When $\delta$ is less than 0.5, environmental factors had a significant effect and effectively reduced the infection rate. The number of infected people showed a decreasing trend. It means that the better environment conditions will inhibit the spread of diseases.

Figure 6 represents the maximum number of infected individuals at different isolation rates. The value range is 0 to 0.9 , and $\delta$ is equal to 0.9 and other data selections are the same as Fig. 5. Through the image, it can be found that the maximum number of infections will decrease with the increase of the isolation rate, and begin tending to balance when it reaches a certain point. The data shows that when $\varepsilon$ is greater than 0.3 , the risk of infection is effectively reduced. For the current data, there is a quarantine inflection whose value is 0.3 . The number of infectious individuals gradually decreases as the isolation rate increases from 0 to 0.3 , and little change after more than 0.3. If the environment changes, we can also calculate a similar quarantine inflection for different parameters. Then based on this result, develop corresponding strategies to control the disease outbreak.

As shown in Fig. 7, according to the data and parameters of the paper [31], and the numbers are given as follow: $S(0)=2.17 \times 10^{8}, I(0)=8, Q(0)=R(0)=$ $0, \beta=0.01, \delta=1, r=0.03, \varepsilon=0.03, \varphi=0.04, d=0.008, \Lambda_{1}=0.01, \Lambda_{2}=$ 


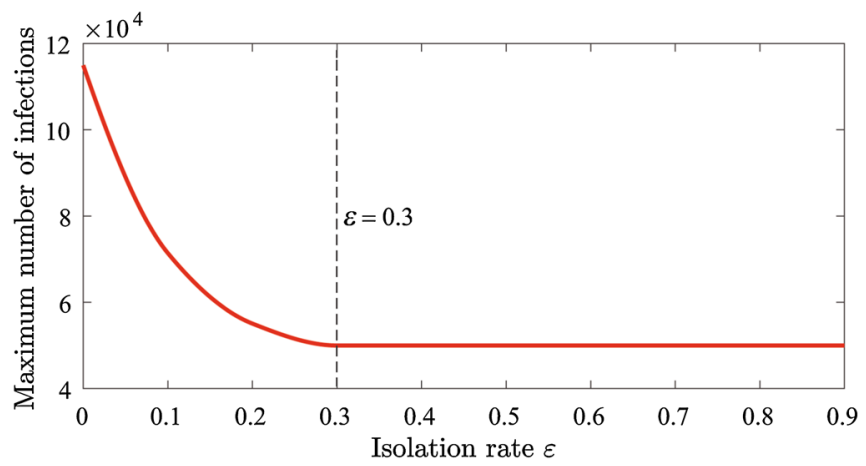

Fig. 6 The change of the maximum value of infection in different isolation rates

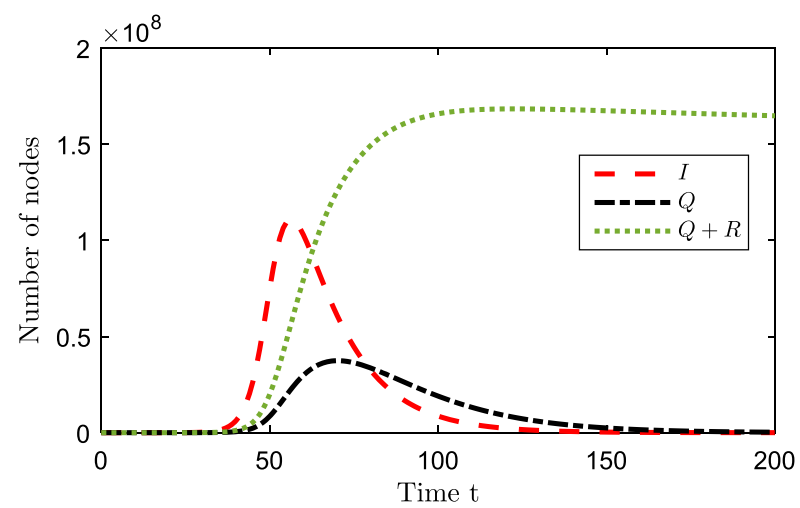

Fig. 7 The parameters is $\delta=1$, time evolution of the number of Infected (I), Quarantined (Q) and total confirmed cases $(\mathrm{Q}+\mathrm{R})$

$0.02, \mu=0.0003$. The figure obtained by simulation is basically the same as the Fig. 2 in the paper [31], and $R_{0}=5.765$ satisfies the condition of the basic reproduction number $R_{0} \in[1.4,6.5]$ in the paper [31]. Next, $\delta$ was adjusted to 0.5 at this point $R_{0}=4.4367$ as shown in Fig. 8. The results show a significant reduction in the number of infected and confirmed cases and a delay in the outbreak of infection. Therefore, a better environment can effectively reduce the risk of early COVID-19 transmission in Brazil.

\section{Conclusion}

In this paper, we investigate the spread of diseases with isolation measures. We propose to consider the model that two different environments mutually impacts. This is different from the previous methodology. The susceptible individuals in the model are not only affected by the infected individuals in the current environment, but also by the infected individuals in other environments. Then we study the existence and 


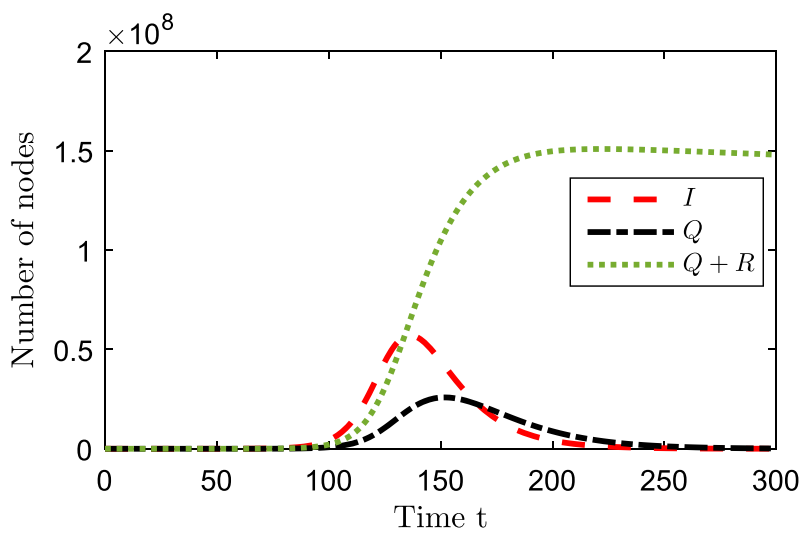

Fig. 8 The parameters is $\delta=0.5$, time evolution of the number of Infected (I), Quarantined (Q) and total confirmed cases $(\mathrm{Q}+\mathrm{R})$

stability of the equilibrium point of the model. Its plausibility is verified by numerical simulations. According to this paper, we propose the following views.

Different environmental factors will affect the spread of current diseases. Different environments will also affect each other. Therefore, when discussing the spread of disease, we believe that it is very important to study the interaction between environments. Isolation measures are significant for studying the interactions between different environments. This is because isolation can effectively limit the mutual flow of people between different environments. The study found that when the isolation level reaches a certain point, the scale of the infection outbreak of the system can be effectively controlled. In reality, we can calculate the critical value according to specific parameters, which is the real meaningful work. Increasing the isolation rate will continuously increase costs, however the impact has been negligible.

In future work, we may expand from the following directions. And consider the impact of multiple diseases and complications. Complications can promote or inhibit the current disease. We propose to find the appropriate solutions in future work.

Funding This work is supported in part by the National Natural Science Foundation of China under (Grant Nos. 62022044 and 61773221), the Jiangsu Natural Science Foundation for Distinguished Young Scholars under (Grant No. BK20190039), and the Scientific Research Foundation of Liaoning Educational Committee of China under (Grant Nos. 2019LNJC13, 2019LNJC11 and 2020LNJC11) and NO.LJKZ0284.

\section{Declarations}

Conflict of interest The authors declare that they have no conflict of interest.

\section{References}

1. World Health Organization. https://covid19.who.int/

2. Wang, J., Dou, J., Sun, M.: Analysis of an SIRS epidemic model with media coverage and logistic variable population. J. Shanxi Norm. Univ. (Nat. Sci. Ed.) 46(6), 52-59 (2018) 
3. Liu, R., Wu, J., Zhu, H.: Media/psychological impact on multiple outbreaks of emerging infectious diseases. Comput. Math. Methods Med. 8(3), 153-164 (2007)

4. Hamer, W.H.: Epidemic disease in England. Lancet 1, 733-739 (1906)

5. Ross, R.: The Prevention of Malaria, pp. 651-686. John Murray, London (1911)

6. Kermack, M., Mckendrick, A.: Contributions to the mathematical theory of epidemics: part I. Proc. R. Soc. Ser. A 115, 700-721 (1927)

7. Jiang, D.Q., Yu, J.J., Ji, C.Y.: Asymptotic behavior of global positive solution to a stochastic SIR model. Math. Comput. Model. 54, 221-232 (2011)

8. Hattaf, K., Lashari, A.A., Louartassi, Y., Yousfi, N.: A delayed SIR epidemic model with general incidence rate. Electron. J. Qual. Theory Differ. Equ. 3, 1-9 (2013)

9. Fan, K.G., Zhang, Y., Gao, S.J., Wei, X.: A class of stochastic delayed SIR epidemic models with generalized nonlinear incidence rate and temporary immunity. Physica A 481, 198-208 (2017)

10. Zhao, S., Stone, L., Gao, D., He, D.: Modelling the large-scale yellow fever outbreak in Luanda. Angola, and the impact of vaccination. PLOS Negl. Trop. Dis. 12(1), e0006158 (2018)

11. Sun, G., Xie, J., Huang, S., Jin, Z., Li, M., Liu, L.: Transmission dynamics of cholera: mathematical modeling and control strategies. Commun. Nonlinear Sci. Numer. Simul. 45, 235-244 (2017)

12. Kim, S., Lee, J., Jung, E.: Mathematical model of transmission dynamics and optimal control strategies for 2009 A/H1N1 influenza in the Republic of Korea. J. Theor. Biol. 412, 74-85 (2017)

13. Grohskopf, L.A., Shay, D.K., Shimabukuro, T.T., Sokolow, L.Z., Keitel, W.A., Bresee, J.S., Cox, N.J.: Prevention and control of seasonal influenza with vaccines: recommendations of the advisory committee on immunization practicesunited states. MMWR Recomm. Rep. 62(7), 1-43 (2013)

14. Wang,M., et al.: Antibody dynamics of 2009 influenza a (h1n1) virus in infected patients and vaccinated people in China. PLoS ONE 6(2), e16809 (2011)

15. Liao, Q., Cowling, B.J., Lam, W.W.T., Fielding, R.: Factors affecting intention to receive and selfreported receipt of 2009 pandemic (h1n1) vaccine in Hong Kong: a longitudinal study. PLoS ONE 6(3), e17713 (2011)

16. Hethcote, H., Ma, Z.E., Liao, S.B.: Effects of quarantine in six endemiec models for infectious diseases. Math. Biosci. 180, 141-160 (2002)

17. Feng, Z., Thieme, H.R.: Recurrent outbreaks of childhood diseases revisited: the impact of isolation. Math. Biosci. 128(1-2), 130 (1995)

18. Ma, Z., Zhou, Y., Wu, J.: Modeling and Dynamics of Infectious Diseases. Higher Education Press, Beijing (2009)

19. Chahrazed, L., Lazhar, R.F.: Stability of a delayed SIQRS model with temporary immunity. Adv. Pure Math. 3(2), 240-245 (2013)

20. Joshi, H., Sharma, R., Prajapati, G.: Global dynamics of an SIQR epidemic model with saturated incidence rate. Asian J. Math. Comput. Res. 21, 156-166 (2017)

21. May, R.M., Allen, P.M.: Stability and complexity in model ecosystems. IEEE Trans. Syst. Man Cybern. 6(12), 887 (1976)

22. Zhang, R., Li, D., Liu, S.: Global analysis of an age-structured SEIR model with immigration of population and nonlinear incidence rate. J. Appl. Anal. Comput. 9(4), 1470-1492 (2019)

23. Liu, Q., Jiang, D., Hayat, T., Alsaedi, A.: Dynamics of a stochastic multigroup SIQR epidemic model with standard incidence rates. J. Frankl. Inst. 356(5), 2960-2993 (2019)

24. Liu, Q., Jiang, D., Hayat, T., Ahmad, B.: Dynamics of a multigroup SIQS epidemic model under regime switching. Stoch. Anal. Appl. 38(5), 769-796 (2020)

25. Iggidr, A., Sallet, G., Souza, M.O.: On the dynamics of a class of multi-group models for vector-borne diseases. J. Math. Anal. Appl. 441(2), 723-743 (2016)

26. Hattaf, K.: A new generalized definition of fractional derivative with non-singular kernel. Computation 8(2), 49-58 (2020)

27. Hattaf, K., Yousfi, N.: Dynamics of SARS-CoV-2 infection model with two modes of transmission and immune response. Math. Biosci. Eng. 17(5), 5326-5340 (2020)

28. Hattaf, K., Mohsen, A.A., Harraq, J., Achtaich, N.: Modeling the dynamics of COVID-19 with carrier effect and environmental contamination. Int. J. Model. Simul. Sci. Comput. 12(3), 2150048-16 (2021)

29. Liu, P., Zhang, L., Xing, Y.: Modelling and stability of a synthetic drugs transmission model with relapse and treatment. J. Appl. Math. Comput. 60, 465-484 (2019). https://doi.org/10.1007/s12190018-01223-0

30. Brauer, F.: Epidemic models with heterogeneous mixing and treatment. Bull. Math. Biol. 70(7), 18691885 (2008) 
31. Crokidakis, N.: Modeling the early evolution of the COVID-19 in Brazil: results from a SusceptibleInfectious-Quarantined-Recovered (SIQR) model. Int. J. Mod. Phys. C 31(10), (2020). https://doi.org/ $10.1142 / \mathrm{S} 0129183120501351$

Publisher's Note Springer Nature remains neutral with regard to jurisdictional claims in published maps and institutional affiliations. 